\title{
(S)-Garner aldehyde derived Baylis-Hillman adduct: a substrate for the synthesis of a lactone ceramide analogue via a sequential Heck reaction
}

\author{
Gulshan Kumar, Sukhbir Kaur, and Vasundhara Singh* \\ Department of Applied Sciences, PEC University of Technology, Chandigarh 160012, India \\ E-mail: vasun7@yahoo.co.in
}

\begin{abstract}
The first, general and efficient route has been developed for the synthesis of $N-((E, 3 S, 4 R)-5$ benzylidene-tetrahydro-4-hydroxy-6-oxo-2H-pyran-3-yl) palmitamide, a lactone ceramide analogue from $(S)$-Garner aldehyde-methyl acrylate derived Baylis-Hillman adduct with anti configuration. Reaction conditions have been optimized for the Heck reaction of iodobenzene on Baylis-Hillman adduct with anti configuration to obtain the protected aromatic ceramide analogue intermediate which subsequently on deprotection and $N$-acylation provided the lactone ceramide analogue. Further, various substituted phenyl iodides have also been employed in the Heck reaction under the optimized conditions for the synthesis of few protected aromatic ceramide analogues intermediates to the test efficacy of the developed methodology.
\end{abstract}

Keywords: Lactone ceramide analogue, Baylis-Hillman reaction, Heck reaction, $(S)$-Garner aldehyde

\section{Introduction}

The Baylis-Hillman ${ }^{1}$ and Heck reactions ${ }^{2}$ are important carbon-carbon bond formation reactions utilized extensively in the synthesis of natural products ${ }^{3,4}$ and their analogues, including $\alpha$ alkylidene- $\beta$-lactams, $\alpha$-methylene- $\gamma$-butyrolactones, frontalin, sarkaomycin, ilmofosine nuciferol, syributins, terpenoids and alkaloids. In the Baylis-Hillman reaction ${ }^{5,6}$ the aldehydes are important substrates and among them the chiral $\alpha$-amino aldehydes ${ }^{7}$ are of special interest as the resulting adducts generate a new stereocenter which can be fine tuned to obtain multifunctional $\alpha$-methylene- $\beta$-hydroxy- $\gamma$-amino acid intermediates to be exploited in asymmetric synthesis. The Heck reaction for arylation of unsubstituted alkenes is controlled by the nature of the substrate, solvent, base, nature of catalyst and its concentration used resulting in variable conditions for obtaining the desired intermediates. ${ }^{8}$ 
Recently, the work on Pd-catalyzed chemical transformations of Baylis-Hillman adducts has been reviewed in literature. ${ }^{9}$ In particular the intermolecular Heck type arylation of achiral Baylis-Hillman adducts has led to $\alpha$-benzyl-substituted $\beta$-keto ester as the major product instead of $\beta$-aryl substituted Baylis-Hillman adducts. ${ }^{10}$ Recently Kim et al. ${ }^{11}$ have successfully optimized condition for intermolecular Heck reaction of achiral aldehyde derived Baylis-Hillman adducts to yield the $\beta$-aryl substituted intermediates as major products.

Sphingolipids, e.g. sphingosine, ceramides, sphingomylein, cerbrosides and gangliosides are important structural and functional components of plasma membrane of essentially all eukaryotic cells and are implicated in the regulation of diverse cellular and physiological processes. ${ }^{12}$ Ceramide [ $N$-acylsphingosine 1a (Figure 1)], a long-chain aliphatic 2-amido-1,3-diol with a $\mathrm{C}(4), \mathrm{C}(5)$-trans double bond, is known to induce apoptotis, ${ }^{13}$ growth arrest ${ }^{14}$ and senescence ${ }^{15}$ in many human cancers, besides regulating many biochemical and cellular responses to stress, such as radiation, oxidative condition, exposure to heat and chemotherapeutic agents. ${ }^{16}$ In addition to the natural ceramides, their analogues have also been evaluated for various biological activities. ${ }^{17}$ In particular, Wascholowski and Giannis ${ }^{18}$ have reported sphingolactones as potent inhibitors of the neutral sphingomyelinase ( $N$-SMase).

Due to the wide spectrum of biological and pharmacological significance of ceramide and its analogues considerable effort has been devoted towards developing efficient methods for their synthesis. ${ }^{19,20}$ Few reports in literature illustrate the use of Baylis-Hillman ${ }^{21}$ and Heck reaction ${ }^{22}$ for the synthesis of sphingolipids and their analogues.

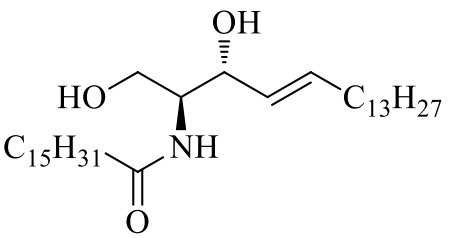

ceramide 1a

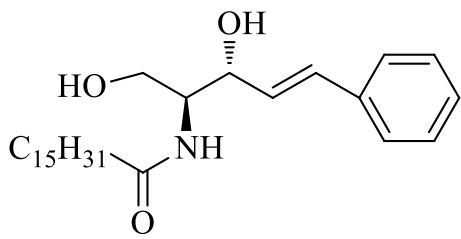

ceramide aromatic analouge $\mathbf{1 b}$

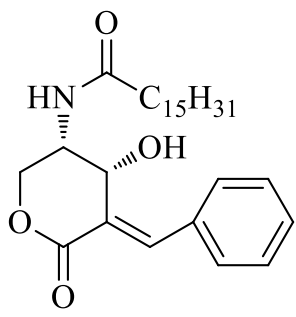

lactone ceramide analouge1c

Figure 1. Ceramide and its analogues.

The configurationally stable Garner aldehyde ${ }^{23,24}$ has received considerable attention as chiral synthon for the stereoselective synthesis of sphingolipids and their derivatives due to its inherent 2-amino-1,3-diol subunit which is the main backbone of sphingolipids. $(S)$-Garner aldehyde-methyl acrylate derived Baylis-Hillman adduct with anti configuration 3a was reported by Drewes et al. ${ }^{21}$ as a potential intermediate for the synthesis of sphingolipid analogue. However, no work has been reported in this direction in literature for further transformations to sphingolipids or their derivatives. 


\section{Results and Discussion}

In continuation to our work ${ }^{25}$ towards the synthesis of sphingolipid and its analogues, we have established the first, efficient and general methodology exploiting the multifunctional molecule 3a for the synthesis of $N$-( $(E, 3 S, 4 R)$-5-benzylidene-tetrahydro-4-hydroxy-6-oxo-2H-pyran-3-yl) palmitamide, a lactone ceramide analogue 1c. The key step involved was $\mathrm{Pd}(\mathrm{OAc})_{2}$ mediated Heck reaction of iodobenzene $\mathbf{4 a}$ on Baylis-Hillman adduct with anti configuration $\mathbf{3 a}$ to obtain the protected ceramide analogue intermediate 6a- $E$ (Scheme 1).

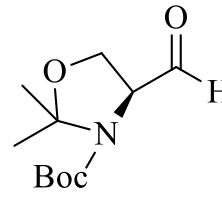

2
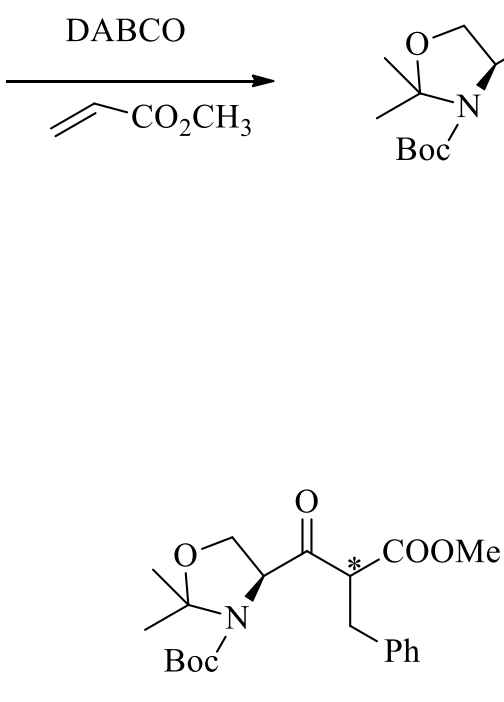

$5 \mathbf{a}$

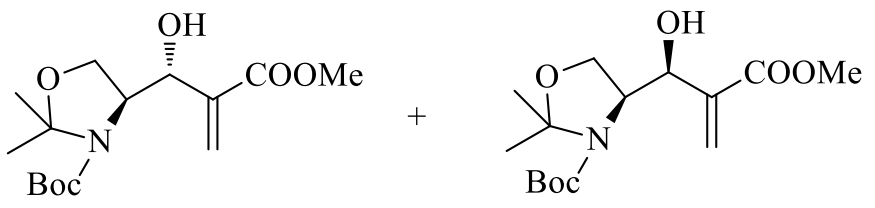

$6: 1$

3a
$\mathrm{PhI} 4 \mathbf{a}, \mathrm{Pd}(\mathrm{OAc})_{2}$

TBAB,KOAc

$\mathrm{CH}_{3} \mathrm{CN}$, reflux, $24 \mathrm{~h}$

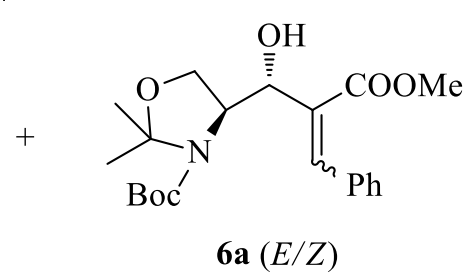

Scheme 1. Baylis Hillman reaction of $(S)$-Garner aldehyde 2 and sequential Heck reaction with $\mathrm{PhI}$ 4a.

The Baylis-Hillman reaction ${ }^{7 \mathrm{~g}}$ of $(S)$-Garner aldehyde $\mathbf{2}$ with methyl acrylate in the presence of DABCO and ultrasound sonication afforded 6:1 diastereomeric mixture of adducts $\mathbf{3 a}$ and $\mathbf{3 b}$ as observed by ${ }^{1} \mathrm{H}$ NMR spectral analysis of crude mixture. The major adduct 3a was separated by column chromatography over silica gel in $73 \%$ yield as a colorless oil and the anti stereochemistry was assigned to $\mathbf{3 a}$ by comparison with spectroscopic data and optical rotation value reported in literature. ${ }^{21,7 \mathrm{~h}}$ The anti stereochemistry of the major adduct may also be rationalized on the basis of the Felkin-Anh open-chain model. ${ }^{26}$

The pure adduct 3a was utilized as a substrate for intermolecular Heck reaction. We first examined the reaction of the adduct $3 \mathbf{a}$ with iodobenzene $4 \mathbf{a}$ to determine the feasibility and optimum $\mathrm{Pd}(\mathrm{OAc})_{2}$ concentration required for the complete consumption of starting adduct 3a and its maximum conversion to the desired Heck product 6a-E/Z (Table 1). 
Table 1. The reaction ${ }^{\mathrm{a}}$ of adduct $\mathbf{3 a}$ with Iodobenzene $\mathbf{4 a}$ under various $\mathrm{Pd}(\mathrm{OAc})_{2}$ concentration

\begin{tabular}{llcccc}
\hline S.No. & Catalyst $(\mathrm{mol} \%)$ & \multicolumn{4}{c}{ Relative composition $(\%)^{\mathrm{b}}$} \\
& & $\mathbf{3 a}$ & $\mathbf{5 a}$ & $\mathbf{6 a -} E$ & $\mathbf{6 a -} \boldsymbol{Z}$ \\
\hline 1 & $\mathrm{Pd}(\mathrm{OAc})_{2}(10)$ & 33 & 30 & 16 & 13 \\
2 & $\mathrm{Pd}(\mathrm{OAc})_{2}(15)$ & 21 & 34 & 20 & 18 \\
3 & $\mathrm{Pd}(\mathrm{OAc})_{2}(20)$ & - & 43 & 27 & 24 \\
4 & $\mathrm{Pd}(\mathrm{OAc})_{2}(25)$ & - & 42 & 28 & 24 \\
\hline
\end{tabular}

3a (1.0 equiv), 4 a (2.0 equiv), TBAB (1.0 equiv), $\mathrm{KOAc}$ (3.0 equiv), mixture in $\mathrm{CH}_{3} \mathrm{CN}$ was refluxed for $24 \mathrm{~h} .{ }^{\text {b }}$ Determined by the ${ }^{1} \mathrm{HNMR}$ spectral analysis of the crude mixture.

The reaction was screened with different concentration of $\mathrm{Pd}(\mathrm{OAc})_{2}$ starting from $10 \mathrm{~mol} \%$ and increasing upto $25 \mathrm{~mol} \%$. It was observed that in the conversion of adduct $3 \mathbf{a}$ to $6 \mathbf{a}-E / Z$, the desired Heck product increases moderately on enhancing the $\operatorname{Pd}(\mathrm{OAc})_{2}$ concentration upto 20 mol\% and beyond which there was no significant effect of increased $\mathrm{Pd}(\mathrm{OAc})_{2}$ concentration on the relative composition (Table 1). The best result was obtained (Table 1, entry 3) when a mixture of 3a (1.0 equiv), $4 \mathbf{a}$ (2.0 equiv), $\mathrm{Pd}(\mathrm{OAc})_{2}(20 \mathrm{~mol} \%)$, TBAB (1.0 equiv.), KOAc (3.0 equiv) in $\mathrm{CH}_{3} \mathrm{CN}$ was refluxed for $24 \mathrm{~h}$. The adduct 3a was consumed completely to give 5a (43\%), 6a- $E$ (27\%) and $\mathbf{6 a}-Z$ in $24 \%$ relative composition as determined by ${ }^{1} \mathrm{H}$ NMR spectral analysis of the crude reaction mixture, wherein the characteristic chemical shift of vinylic proton of $\mathbf{6 a}-E$ was observed as a singlet at $7.79 \mathrm{ppm}$ and for $\mathbf{6 b}-Z$ isomer at $7.01 \mathrm{ppm}$, while the benzylic $-\mathrm{CH}_{2}$ of $\alpha$-benzyl- $\beta$-keto ester $\mathbf{5 a}$ appeared at $3.17 \mathrm{ppm}$ as a multiplet. Purification by column chromatography over silica gel, eluting with hexane:EtOAc/89:11, yielded $\beta$-keto ester 5a $(36 \%)$ as a white solid. Further elution with hexane:EtOAc/86:14 afforded 6a- $E(21 \%)$ and 6b-Z (18\%) as colorless oils.

Under these optimized conditions the Heck reaction was carried out with adduct 3a and various aryl iodides $\mathbf{4 b}$-d to obtain the desired Heck products $\mathbf{6 b}$-d and corresponding $\beta$-keto esters 5b-d as shown in Scheme 2 and results are summarized in Table 2.

$$
\begin{aligned}
& \begin{aligned}
\mathbf{3 a}+\operatorname{ArI} & \frac{\operatorname{Pd}(\mathrm{OAc})_{2}, \mathrm{TBAB}}{\text { 4b-d }} \begin{array}{l}
\mathrm{KOAc}, \mathrm{CH}_{3} \mathrm{CN} \\
\text { Reflux, } 24 \mathrm{~h}
\end{array}
\end{aligned} \\
& \text { 4b: } \mathrm{Ar}=4-\mathrm{MeC}_{6} \mathrm{H}_{4} \\
& \text { 4c: } \mathrm{Ar}=4-\mathrm{MeOC}_{6} \mathrm{H}_{4} \\
& \text { 4d: } \mathrm{Ar}=4-\mathrm{MeCOC}_{6} \mathrm{H}_{4}
\end{aligned}
$$

Scheme 2. Heck reaction of adduct 3a with selected aryl halides $\mathbf{4 b - d . ~}$ 
Table 2. Heck reaction of adduct $\mathbf{3 a}$ with selected aryl iodides $\mathbf{4 b - \mathbf { d } ^ { \mathrm { a } }}$

\begin{tabular}{llll}
\hline S.No. & Substrates & \multicolumn{1}{c}{ Relative composition $(\%)^{\mathrm{b}}$} & \multicolumn{1}{c}{${\text { Yield }(\%)^{\mathrm{c}, \mathrm{d}}}^{\mathrm{b}}$} \\
\hline 1 & $\mathbf{3 a}+\mathbf{4 b}$ & $\mathbf{3 a}(0) / \mathbf{5 b}(49) / \mathbf{6 b}-E(24) / \mathbf{6 b}-Z(22)$ & $\mathbf{5 b}(41) / \mathbf{6 b}(37)$ \\
2 & $\mathbf{3 a}+\mathbf{4 c}$ & $\mathbf{3 a}(0) / \mathbf{5 c}(48) / \mathbf{6 c}-E(25) / \mathbf{6 c}-Z(23)$ & $\mathbf{5 c}(43) / \mathbf{6 c}(41)$ \\
3 & $\mathbf{3 a}+\mathbf{4 d}$ & $\mathbf{3 a}(40) / \mathbf{5 d}(30) / \mathbf{6 d}-E(14) / \mathbf{6 d}-Z(12)$ & $\mathbf{3 a}(36) / \mathbf{5 d}(26) / \mathbf{6 d}(21)$ \\
\hline
\end{tabular}

${ }^{a}$ Conditions: compound 3a (1.0 equiv), aryl Iodides 4a-d (2.0 equiv), $\mathrm{Pd}(\mathrm{OAc})_{2}(20 \mathrm{~mol} \%)$, TBAB(1.0 equiv), KOAc (3.0 equiv), $\mathrm{CH}_{3} \mathrm{CN}$, reflux, $24 \mathrm{~h}$. ${ }^{\mathrm{b}}$ Determined by the ${ }^{1} \mathrm{H}$ NMR spectral analysis of the crude mixture. ${ }^{\mathrm{c}}$ Isolated yield. ${ }^{\mathrm{d}} \mathrm{E} / \mathrm{Z}$ mixture not separated.

It has been observed that the adduct 3a was completely consumed except when $p$ iodoacetophenone 4d (Table 2, entry 3) was used as the aryl halide, under the optimized conditions. The relative composition of products was again determined by ${ }^{1} \mathrm{H}$ NMR spectral analysis of respective crude mixtures. It has been observed from ${ }^{1} \mathrm{H}$ NMR spectra that the isomer $E$ and $Z$ for compounds 6a-d are obtained in approximately 1:1 ratio as explained by Kumareswaran and Vankar. ${ }^{10 a}$ The $\beta$-keto esters 5b-d were isolated in $26-43 \%$ yield and the desired Heck products were isolated in $21-41 \%$ yield. The $E$ and $Z$ isomers of $\mathbf{6 b - d}$ were not separated. All compounds were characterized by NMR, IR, and mass spectroscopy.

The $N$-Boc and oxazolidine groups of pure $6 \mathbf{a}-E$ were removed under acidic condition using $1 \mathrm{M} \mathrm{HCl} / \mathrm{MeOH}$ followed by $N$-acylation ${ }^{27}$ using $p$-nitrophenyl palmitate, $\mathrm{Et}_{3} \mathrm{~N}$, DMAP (cat.) in dry THF to afford crude lactone ceramide analogue 1c as the major product instead of the expected open chain ceramide analogue. The lactonisation may have occurred due to the intramolecular condensation of primary hydroxyl group of the sphingoid backbone with $-\mathrm{COOH}$ group generated during the deprotection step of intermediate $6 \mathbf{a}-E$ or by the direct attack of the primary hydroxyl group to the ester group, before its hydrolysis, with subsequent elimination of methanol. The mixture was purified by column chromatography over silica gel to obtain the product 1c in 54\% yield (Scheme 3). The title compound 1c was characterized by NMR, IR and mass spectroscopy. The characteristic peaks for 1c were observed for the carbonyl of lactone at $1732 \mathrm{~cm}^{-1}$ in the IR spectrum and at $\delta 4.30-4.31(\mathrm{~d}, J=6.2 \mathrm{~Hz}, 1 \mathrm{H}, \underline{\mathrm{HCHOH}})$ and $4.40-4.45(\mathrm{~m}$, $2 \mathrm{H}, \mathrm{HC} \underline{\mathrm{HOH}},-\mathrm{C} \underline{\mathrm{H}}-\mathrm{N}$ ) in the ${ }^{1} \mathrm{H}$ NMR spectrum, which showed downfield shift of these protons as compared to that expected in the corresponding acyclic ceramide analogue. In the ${ }^{13} \mathrm{C} \mathrm{NMR}$ spectrum, the peaks at $\delta 45.6$ and 164.9 were observed for $-\mathrm{C}-\mathrm{N}$ and for lactone group respectively, which further confirmed the formation of lactone ceramide analogue 1c. 
<smiles>COC(=O)/C(=C/c1ccccc1)[C@@H](O)[C@H]1COC(C)(C)N1C(=O)OCc1ccccc1</smiles>

6a $(E)$

$$
\begin{array}{r}
\underset{\text { 2. } p-\mathrm{NO}_{2} \mathrm{C}_{6} \mathrm{H}_{4} \mathrm{OCOC}_{15} \mathrm{H}_{31}}{\mathrm{Et}} \\
\underset{\mathrm{Et}_{3} \mathrm{~N}, \mathrm{DMAP} \text { (cat.) THF }}{\longrightarrow}
\end{array}
$$

1. $\mathrm{HCl}, \mathrm{MeOH}$

X 2. $p-\mathrm{NO}_{2} \mathrm{C}_{6} \mathrm{H}_{4} \mathrm{OCOC}{ }_{15} \mathrm{H}_{31}$

$\checkmark$

$\mathrm{Et}_{3} \mathrm{~N}, \mathrm{DMAP}$ (cat.), THF

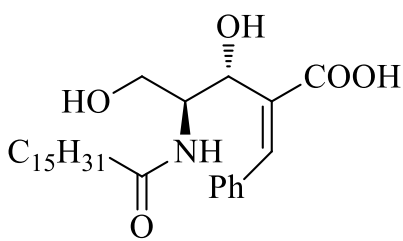

Scheme 3. Synthesis of lactone ceramide analogue 1c.

\section{Conclusions}

In summary: we have developed a methodology for the efficient synthesis of lactone ceramide analogues from chiral precursors, accessible via Heck reaction on adduct 3a. To the best of our knowledge this is the first approach for the Heck reaction on chiral aldehyde derived BaylisHillman adduct and its use as a chiral synthon for synthesis of $N-((E, 3 S, 4 R)-5$-benzylidenetetrahydro-4-hydroxy-6-oxo-2H-pyran-3-yl)palmitamide, a lactone ceramide analogue 1c. This general methodology could be utilized for synthesis of lactone ceramide substituted aromatic analogues which could be evaluated for biological activities.

\section{Experimental Section}

General. ${ }^{1} \mathrm{H}$ and ${ }^{13} \mathrm{C}$ NMR spectra were recorded on a Bruker Avance II $400 \mathrm{MHz}$ spectrometer at 400 and $100 \mathrm{MHz}$ respectively using TMS as internal standard in $\mathrm{CDCl} 3$. IR spectra were recorded using a Perkin-Elmer model RX 1 FT-IR spectrometer. Mass spectra were recorded on Waters Micromass q-Tof Micro spectrometer. Elemental analyses were performed using automatic Perkin Elmer $2400 \mathrm{CHN}$ elemental analyzer. Optical rotations (in degrees) were recorded on Autopol-III polarimeter. The Baylis-Hillman reaction was sonicated in an ultrasonic cleaner $(40 \pm 5 \mathrm{kHz})$. Column chromatography was conducted on Silica gel 100-200 mesh (E- 
Merck) using ethyl acetate and hexane as eluant. All reactions were carried out using oven dried glassware. All solvents were dried as reported in literature prior to use.

(S)-tert-Butyl 4-((R)-2-(methoxycarbonyl)-1-hydroxyallyl)-2,2-dimethyloxazolidine-3carboxylate (3a). A mixture of Garner aldehyde $2(1.2 \mathrm{~g}, 5.24 \mathrm{mmol})$, methyl acrylate $(0.677 \mathrm{~g}$, $7.86 \mathrm{mmol})$, and DABCO $(0.588 \mathrm{~g}, 5.24 \mathrm{mmol})$ was sonicated for $40 \mathrm{~h}$ till there was no further change in reaction mixture composition as monitored by TLC. The ultrasound bath temperature was constantly monitored and maintained at $30-40{ }^{\circ} \mathrm{C}$ during the reaction, through ice addition. After $40 \mathrm{~h}$, the mixture was evaporated under reduced pressure in order to remove excess of methyl acrylate. The residue was diluted with dichloromethane $(30 \mathrm{ml})$, washed successively with cold $5 \%$ aqueous $\mathrm{HCl}(2 \mathrm{X} 15 \mathrm{ml})$, saturated $\mathrm{NaHCO}_{3}(20 \mathrm{ml})$, brine $(20 \mathrm{ml})$ and dried over $\mathrm{Na}_{2} \mathrm{SO}_{4}$. After filtration the solvent was removed under reduced pressure. The ${ }^{1} \mathrm{H}$ NMR analysis of crude mixture indicated the formation of Baylis-Hillman adducts 3a:3b in 6:1 diastereomeric ratio. The residue was purified by column chromatography over silica gel, eluting with hexane:EtOAc $(85: 15)$ to afford pure major adduct $\mathbf{3 a}(1.20 \mathrm{~g}, 73 \%)$ as a colorless oil. $[\alpha]_{\mathrm{D}}{ }^{25}-1.8$ (c 1, $\mathrm{CHCl}_{3}$ ); IR (neat) $v_{\max }\left(\mathrm{cm}^{-1}\right) 3464,1715,1696,1389,1261,1165,1096 ;{ }^{1} \mathrm{H}$ NMR (400 $\left.\mathrm{MHz} \mathrm{CDCl}_{3}\right): \delta 1.45-1.55(\mathrm{~m}, 15 \mathrm{H}), 3.79$ (s, 3H), 3.92 (br s, $\left.1 \mathrm{H}\right), 4.14-4.25(\mathrm{~m}, 2 \mathrm{H}), 4.48-4.62$ $(\mathrm{m}, 2 \mathrm{H}), 5.79$ (br s, 1H), 6.25 (br s, 1H) ppm; $\left.{ }^{13} \mathrm{C} \mathrm{NMR} \mathrm{(100} \mathrm{MHz,} \mathrm{CDCl}_{3}\right): \delta 24.2,27.0,28.3$, $51.8,61.7,65.0,74.3,80.6,94.1,126.9,140.2,153.8,166.9 \mathrm{ppm}$. The spectroscopic data of the major adduct $3 \mathbf{a}$ were consistent with those reported in literature. ${ }^{20}$

\section{General procedure for the Heck coupling of $(S)$-Garner aldehyde-methyl acrylate derived Baylis-Hillman adducts 3 a with various aryl halides (4a-d)}

A stirred solution of $(S)$-Garner aldehyde-methyl acrylate derived Baylis-Hillman adduct 3a (1.0 equiv), aryl halides $4 \mathbf{a}-\mathbf{d}$ (2.0 equiv), $\mathrm{Pd}(\mathrm{OAc})_{2}(20 \mathrm{~mol} \%), \mathrm{KOAc}$ (3.0 equiv), TBAB (1.0 equiv) in dry $\mathrm{CH}_{3} \mathrm{CN}(8-10 \mathrm{ml})$ was heated to reflux for $24 \mathrm{~h}$ under nitrogen atmosphere. The reaction mixture was allowed to warm to room temperature followed by solvent removal under reduced pressure. The residue was diluted with water and extracted with diethyl ether $(5 \mathrm{x} 10 \mathrm{ml})$. The combined organic layers were dried over anhydrous $\mathrm{Na}_{2} \mathrm{SO}_{4}$, filtered and the crude product was passed through a small pad of celite. The solvent was evaporated under reduced pressure and ${ }^{1} \mathrm{H}$ NMR spectra was recorded to determine conversion ratio of the products. The column chromatographic separation over silica gel, eluting with hexane/EtOAc (89:11-72:28) yielded the $\beta$-keto esters 5a-d in $26-43 \%$ as a white solids and desired Heck products 6a-d (E/Z) in $21-41 \%$ as colorless oils.

Compound (5a) (diastereomeric mixture). White solid; $36 \%$ yield; mp 120-122 ${ }^{\circ} \mathrm{C}$; $[\alpha]_{\mathrm{D}}{ }^{25}+$ 2.6 (c 1, $\left.\mathrm{CHCl}_{3}\right)$; IR (KBr) $v_{\max }\left(\mathrm{cm}^{-1}\right) 3012,2931,1741,1687,1617,1365,1262,1160,1081 ;{ }^{1} \mathrm{H}$ NMR (400 MHz, $\left.\mathrm{CDCl}_{3}\right): \delta 1.40-1.62(\mathrm{~m}, 15 \mathrm{H}), 3.16-3.18(\mathrm{~m}, 2 \mathrm{H}), 3.66(\mathrm{~s}, 3 \mathrm{H}), 3.72-3.76(\mathrm{~m}$, $1 \mathrm{H}), 3.91-4.07(\mathrm{~m}, 2 \mathrm{H}), 4.56-4.63(\mathrm{~m}, 1 \mathrm{H}), 7.16-7.26(\mathrm{~m}, 5 \mathrm{H}) \mathrm{ppm} ;{ }^{13} \mathrm{C} \mathrm{NMR}(100 \mathrm{MHz}$, $\left.\mathrm{CDCl}_{3}\right): \delta 24.7,25.2,28.2,29.7,34.2,52.5,52.6,57.1,57.3,64.3,64.5,65.0,65.2,80.7,81.1$, $94.7,95.4,126.7,126.9,128.5,128.6,128.9,137.9,138.2,151.4,152.4,168.5,168.7,200.0$, 
201.7 ppm. MS, $m / z(\%)=392.1(\mathrm{M}+1,8)^{+}, 414.1(\mathrm{M}+\mathrm{Na}, 100)^{+}$; Anal. Calcd. for $\mathrm{C}_{21} \mathrm{H}_{29} \mathrm{NO}_{6}$ : C, 64.43; H, 7.46; N, 3.57\%. Found: C, 64.36; H, 7.40; N, 3.52\%.

(S)-tert-Butyl

4-((R,E)-2-(methoxycarbonyl)-1-hydroxy-3-phenylallyl)-2,2-

dimethyloxazolidine-3-carboxylate (6a- $\boldsymbol{E})$. Colorless oil; $21 \%$ yield; $[\alpha]_{\mathrm{D}}^{25}-7.5\left(\boldsymbol{c} 1, \mathrm{CHCl}_{3}\right)$; IR (neat) $v_{\max }\left(\mathrm{cm}^{-1}\right) 3460,1712,1672,1389,1261,1165,1096 ;{ }^{1} \mathrm{H} \mathrm{NMR}\left(400 \mathrm{MHz}, \mathrm{CDCl}_{3}\right): \delta$ 1.44-1.60 (m, 15H), 3.79-3.87 (m, 1H), 3.88 (s, 3H), 4.07 (br s, 1H), 4.17 (d, J = 9.2 Hz, 1H), 4.29-4.32 (m, 1H), 4.59 (d, J=9.7 Hz, 1H), 7.26-7.39 (m, 5H), $7.76(\mathrm{~s}, 1 \mathrm{H}) \mathrm{ppm} ;{ }^{13} \mathrm{C}$ NMR (100 $\left.\mathrm{MHz}_{\mathrm{CDCl}}\right): \delta 24.0,26.5,28.3,51.0,60.1,65.0,69.4,79.9,93.6,128.3,128.6,128.8,131.3$, 134.8, 141.6, 152.8, 168.7 ppm. MS, $m / z(\%)=414.4(\mathrm{M}+\mathrm{Na}, 76)^{+}, 415.4(\mathrm{M}+1+\mathrm{Na}, 20)^{+}$, $374.4(\mathrm{M}-\mathrm{OH}, 11)^{+}, 314.4\left(\mathrm{M}^{+}-\mathrm{C}_{6} \mathrm{H}_{5}, 6\right)^{+}$; Anal. Calcd for $\mathrm{C}_{21} \mathrm{H}_{29} \mathrm{NO}_{6}: \mathrm{C}, 64.43 ; \mathrm{H}, 7.46 ; \mathrm{N}$, $3.57 \%$. Found: C, 64.37; H, 7.39; N, 3.54\%.

(S)-tert-Butyl

4-((R,Z)-2-(methoxycarbonyl)-1-hydroxy-3-phenylallyl)-2,2-

dimethyloxazolidine-3-carboxylate (6a- $Z$ ). colorless oil; $18 \%$ yield; $[\alpha]_{\mathrm{D}}{ }^{25}-8.1$ (c $\left.1, \mathrm{CHCl}_{3}\right)$; IR (neat) $v_{\max }\left(\mathrm{cm}^{-1}\right) \quad 3464,1715,1685,1391,1265,1156,1085 ;{ }^{1} \mathrm{H}$ NMR $\left(400 \mathrm{MHz}, \mathrm{CDCl}_{3}\right): \delta$ $1.42-1.61(\mathrm{~m}, 15 \mathrm{H}), 3.64(\mathrm{~s}, 3 \mathrm{H}), 3.79-3.85(\mathrm{~m}, 1 \mathrm{H}), 3.97$ (br s, $1 \mathrm{H}), 4.18(\mathrm{dd}, J=1.2,9.5 \mathrm{~Hz}$, $1 \mathrm{H}), 431-4.32(\mathrm{~m}, 1 \mathrm{H}), 4.59(\mathrm{~d}, J=9.7 \mathrm{~Hz}, 1 \mathrm{H}), 7.05(\mathrm{~s}, 1 \mathrm{H}), 7.24-7.37(\mathrm{~m}, 5 \mathrm{H}) \mathrm{ppm} ;{ }^{13} \mathrm{C} \mathrm{NMR}$ $\left(100 \mathrm{MHz}_{\mathrm{CDCl}}\right): \delta$ 25.2, 27.2, 29.2, 51.0, 61.5, 65.0, 69.1, 78.9, 92.6, 127.1, 127.9, 129.5, $132.0,134.8,140.6,151.8,168.3 \mathrm{ppm}$. MS, $m / z(\%)=414.4(\mathrm{M}+\mathrm{Na}, 78)^{+}, 374.4(\mathrm{M}-\mathrm{OH}$, $12)^{+,} 314.4\left(\mathrm{M}-\mathrm{C}_{6} \mathrm{H}_{5}, 5\right)^{+}$; Anal. Calcd for $\mathrm{C}_{21} \mathrm{H}_{29} \mathrm{NO}_{6}: \mathrm{C}, 64.43 ; \mathrm{H}, 7.46 ; \mathrm{N}, 3.57 \%$. Found: $\mathrm{C}$, $64.38 ; \mathrm{H}, 7.39 ; \mathrm{N}, 3.55 \%$.

Compound (5b) (diastereomeric mixture). white solid; $41 \%$ yield; mp $151-153{ }^{\circ} \mathrm{C}$; $[\alpha]_{\mathrm{D}}{ }^{25}+2.9$ (c 1.1, $\left.\mathrm{CHCl}_{3}\right)$; IR (KBr) $v_{\max }\left(\mathrm{cm}^{-1}\right) 3032,2935,1745,1625,1695,1610,1384,1254,1168$, 1067; ${ }^{1} \mathrm{H}$ NMR (400 MHz, $\left.\mathrm{CDCl}_{3}\right): \delta$ 1.43-1.62 (m, 15H), $2.29(\mathrm{~s}, 3 \mathrm{H}), 3.13-3.18(\mathrm{~m}, 2 \mathrm{H}), 3.66$ (s, 3H), 3.71-3.79 (m, 1H), 3.89-4.10 (m, 2H), 4.56-4.63 (m, 1H), 7.04-7.07 (m, 4H) ppm; ${ }^{13} \mathrm{C}$ NMR (100 MHz, $\left.\mathrm{CDCl}_{3}\right): \delta$ 23.6, 24.8, 25.7, 26.0, 28.3, 29.3, 29.7, 33.8, 52.4, 57.2, 57.4, 64.6, 64.8, 65.0, 65.2, 80.8, 81.1, 94.9, 95.4, 128.7, 129.2, 129.3, 136.4, 151.8, 168.8, 200.2 ppm. MS, $m / z(\%)=428.5(\mathrm{M}+\mathrm{Na}, 100)^{+}$; Anal. Calcd for $\mathrm{C}_{22} \mathrm{H}_{31} \mathrm{NO}_{6}: \mathrm{C}, 65.16 ; \mathrm{H}, 7.70 ; \mathrm{N}, 3.45 \%$. Found: C, 65.10; H, 7.65; N, 3.41\%.

(S)-tert-Butyl 4-((R)-2-(methoxycarbonyl)-1-hydroxy-3-p-tolylallyl)-2,2-dimethyloxazolidine-3-carboxylate $(\mathbf{6 b}-\boldsymbol{E} / \boldsymbol{Z})$. colorless oil; 37 yield\%; $\alpha]_{\mathrm{D}}{ }^{25}-7.3\left(c 1, \mathrm{CHCl}_{3}\right)$; IR (neat) $v_{\max }\left(\mathrm{cm}^{-1}\right)$ 3470, 2903, 1705, 1370, 1261, 1177, 1076; ${ }^{1} \mathrm{H}$ NMR (400 MHz, $\left.\mathrm{CDCl}_{3}\right): \delta 1.43-1.62$ $(\mathrm{m}, 15 \mathrm{H}), 2.34(\mathrm{~s}, 3 \mathrm{H}), 3.66(\mathrm{~s}, 1.44 \mathrm{H}), 3.78-3.84(\mathrm{~m}, 1 \mathrm{H}), 3.88(\mathrm{~s}, 1.56 \mathrm{H}), 3.97(\mathrm{br} \mathrm{s}, 1 \mathrm{H}), 4.17$ $(\mathrm{dd}, J=1.6,7.9 \mathrm{~Hz}, 1 \mathrm{H}), 428-4.32(\mathrm{~m}, 1 \mathrm{H}), 4.62(\mathrm{~d}, J=9.7 \mathrm{~Hz}, 1 \mathrm{H}), 7.0(\mathrm{~s}, 0.48 \mathrm{H}), 7.10-7.25$ $(\mathrm{m}, 4 \mathrm{H}), 7.72(\mathrm{~s}, 0.52 \mathrm{H}) \mathrm{ppm} ;{ }^{13} \mathrm{C} \mathrm{NMR}\left(100 \mathrm{MHz}, \mathrm{CDCl}_{3}\right): \delta$ 23.3, 25.5, 27.2, 29.9, 50.8, 51.0, 59.0, 64.0, 68.4, 78.9, 92.5, 127.3, 127.9, 128.7, 130.8, 131.1, 137.6, 140.8, 151.8, 167.8 ppm; MS, $m / z(\%)=428.5(\mathrm{M}+\mathrm{Na}, 100)^{+}, 388.5\left(\mathrm{M}^{+}-\mathrm{OH}, 20\right)^{+}$; Anal. Calcd for $\mathrm{C}_{22} \mathrm{H}_{31} \mathrm{~N} \mathrm{O}_{6}: \mathrm{C}$, 65.12; H, 7.70; N, 3.45\%. Found: C, 65.09; H, 7.66; N, 3.42\%.

Compound (5c) (diastereomeric mixture). White solid; 43\% yield; mp 171-174 ${ }^{\circ} \mathrm{C} ;[\alpha]_{\mathrm{D}}{ }^{25}$ +2.7 (c 1, $\left.\mathrm{CHCl}_{3}\right)$; IR (KBr) v $v_{\max }\left(\mathrm{cm}^{-1}\right) 3030,2955,1748,1675,1620,1364,1275,1162,1045$; ${ }^{1} \mathrm{H}$ NMR (400 MHz, $\left.\mathrm{CDCl}_{3}\right): \delta 1.42-1.63(\mathrm{~m}, 15 \mathrm{H}), 3.11-3.17(\mathrm{~m}, 2 \mathrm{H}), 3.66(\mathrm{~s}, 3 \mathrm{H}), 3.71-3.80$ 
(m, 4H), 3.89-4.08 (m, 2H), 4.54-4.61 (m, 1H), 6.79-6.81 (m, 2H), 7.07-7.13 (m, 2H) ppm; ${ }^{13} \mathrm{C}$ NMR (100 MHz, $\left.\mathrm{CDCl}_{3}\right): \delta$ 23.6, 24.7, 26.0, 28.2, 29.7, 33.1, 33.6, 52.4, 52.5, 55.2, 57.6, 57.9, 64.4, 64.6, 65.0, 65.2, 80.7, 81.1, 94.7, 95.4, 113.9, 114.0, 129.8, 130.0, 130.3, 151.2, 151.4, $158.4,168.6,168.9,200.2,201.8 \mathrm{ppm}$. MS, $m / z(\%)=444.4(\mathrm{M}+\mathrm{Na}, 100)^{+}$; Anal. Calcd for $\mathrm{C}_{22} \mathrm{H}_{31} \mathrm{NO}_{7}$ : C, 62.69; H, 7.41; N, 3.32\%. Found: C, 62.62; H, 7.36; N, 3.29\%.

(S)-tert-Butyl 4-((R)-2-(methoxycarbonyl)-1-hydroxy-3-(4-methoxyphenylallyl)-2,2dimethyloxazolidine-3-carboxylate (6c-E/Z). Viscous oil; $41 \%$ yield; $\left.[\alpha]_{\mathrm{D}}^{25}-7.1(c) 1, \mathrm{CHCl}_{3}\right)$; IR (neat) $v_{\max }\left(\mathrm{cm}^{-1}\right) 3460,2931,1720,1375,1261,1154,1093 ;{ }^{1} \mathrm{H}$ NMR (400 MHz, CDCl 3 ): $\delta$ 1.42-1.61 (m, 15H), $3.68(\mathrm{~s}, 1.44 \mathrm{H}), 3.80-3.84(\mathrm{~m}, 4 \mathrm{H}), 3.88(\mathrm{~s}, 1.56 \mathrm{H}), 3.97$ (br s, $1 \mathrm{H}), 4.18$ $(\mathrm{dd}, J=1.3,9.4 \mathrm{~Hz}, 1 \mathrm{H}), 429-4.33(\mathrm{~m}, 1 \mathrm{H}), 4.65(\mathrm{~d}, J=9.7 \mathrm{~Hz}, 1 \mathrm{H}), 6.82-6.90(\mathrm{~m}, 2 \mathrm{H}), 6.96$ (s, $0.48 \mathrm{H}), 7.22-7.30(\mathrm{~m}, 2 \mathrm{H}), 7.70(\mathrm{~s}, 0.52 \mathrm{H}) \mathrm{ppm} ;{ }^{13} \mathrm{C} \mathrm{NMR}\left(100 \mathrm{MHz}, \mathrm{CDCl}_{3}\right): \delta$ 24.1, 26.6, 28.4, 29.7, 51.8, 55.2, 55.3, 60.1, 65.1, 69.5, 80.0, 80.1, 93.6, 114.1, 116.1, 127.1, 129.6, 130.5, 141.6, 152.8, 159.6, 159.9, $168.9 \mathrm{ppm}$. MS, $m / z(\%)=444.4(\mathrm{M}+\mathrm{Na}, 100)^{+}$; Anal. Calcd for $\mathrm{C}_{22} \mathrm{H}_{31} \mathrm{NO}_{7}$ : C, 62.69; H, 7.41; N, 3.32\%. Found: C, 62.61; H, 7.37; N, 3.28\%.

Compound (5d) (diastereomeric mixture). White solid; 26 yield\%; mp 195-197 ${ }^{\circ} \mathrm{C}$; $[\alpha]_{\mathrm{D}}{ }^{25}$ +3.1 (c 1, $\left.\mathrm{CHCl}_{3}\right)$; IR (KBr) $v_{\max }\left(\mathrm{cm}^{-1}\right) 3052,2934,1751,1681,1607,1387,1269,1096 ;{ }^{1} \mathrm{H}$ NMR (400 MHz, $\left.\mathrm{CDCl}_{3}\right): \delta 1.41-1.60(\mathrm{~m}, 15 \mathrm{H}), 2.57(\mathrm{~s}, 3 \mathrm{H}), 3.22-3.24(\mathrm{~m}, 2 \mathrm{H}), 3.66(\mathrm{~s}, 3 \mathrm{H})$, 3.71-3.87 (m, 1H), 3.89-4.06 (m, 2H), 4.49-4.63 (m, 1H), 7.28-7.31 (m, 2H), 7.85-7.87 (m, 2H) ppm; ${ }^{13} \mathrm{C} \mathrm{NMR}\left(100 \mathrm{MHz}, \mathrm{CDCl}_{3}\right): \delta$ 24.2, 25.5, 27.9, 28.6, 30.9, 33.0, 51.5, 57.6, 57.9, 64.7, 64.8, 65.1, 65.3, 80.7, 81.1, 94.7, 95.4, 127.7, 128.1, 129.2, 136.4, 144.5, 152.1, 168.3, 196.7, $200.1 \mathrm{ppm} ; \mathrm{MS}, \mathrm{m} / z(\%)=456.4(\mathrm{M}+\mathrm{Na}, 95)^{+}$; Anal. Calcd for $\mathrm{C}_{23} \mathrm{H}_{31} \mathrm{NO}_{7}$ : C, 63.72; $\mathrm{H}, 7.20$; N, 3.23\%. Found: C, 63.67; H, 7.16; N, 3.18\%.

(S)-tert-Butyl 4-((R)-2-(methoxycarbonyl)-3-(4-acetylphenyl)-1-hydroxyallyl)-2,2dimethyloxazolidine-3-carboxylate (6d-E/Z). Colorless viscous oil; 21\% yield; $[\alpha]_{\mathrm{D}}{ }^{25}-6.8(c$ $\left.1, \mathrm{CHCl}_{3}\right)$; IR (neat) $v_{\max }\left(\mathrm{cm}^{-1}\right) 3467,2954,1715,1690,1389,1272,1165,1059 ;{ }^{1} \mathrm{H}$ NMR (400 $\left.\mathrm{MHz}, \mathrm{CDCl}_{3}\right) \delta 1.41-1.60(\mathrm{~m}, 15 \mathrm{H}), 2.60(\mathrm{~s}, 3 \mathrm{H}), 3.62(\mathrm{~s}, 1.44 \mathrm{H}), 3.85(\mathrm{dd}, J=5.4,9.0 \mathrm{~Hz}, 1 \mathrm{H})$, $3.90(\mathrm{~s}, 1.56 \mathrm{H}), 4.01$ (br s, 1H), $4.16(\mathrm{dd}, J=2.9,9.2 \mathrm{~Hz}, 1 \mathrm{H}), 431-4.33(\mathrm{~m}, 1 \mathrm{H}), 4.51(\mathrm{~d}, J=$ $9.6 \mathrm{~Hz}, 1 \mathrm{H}), 7.07(\mathrm{~s}, 0.48 \mathrm{H}), 7.31-7.44(\mathrm{~m}, 2 \mathrm{H}), 7.74(\mathrm{~s}, 0.52 \mathrm{H}), 7.89-7.97(\mathrm{~m}, 2 \mathrm{H}) \mathrm{ppm} ;{ }^{13} \mathrm{C}$ NMR $\left(100 \mathrm{MHz}, \mathrm{CDCl}_{3}\right) \delta$ 23.9, 26.6, 28.4, 29.3, 29.5, 29.7, 52.0, 60.1, 64.9, 69.5, 80.1, 93.6, 128.6, 129.0, $133.0136 .6,139.6,140.0,152.9,168.3,197.4 \mathrm{ppm} . \mathrm{MS}, m / z(\%)=456.6(\mathrm{M}+\mathrm{Na}$, 100)+; Anal. Calcd for $\mathrm{C}_{23} \mathrm{H}_{31} \mathrm{NO}_{7}$ : C, 63.72; H, 7.20; N, 3.23\%. Found: C, 63.65; H, 7.17; N, $3.19 \%$.

$N$-((E,3S,4R)-5-Benzylidene-tetrahydro-4-hydroxy-6-oxo-2H-pyran-3-yl)palmitamide (1c). To a solution of $\mathbf{5 a}-\boldsymbol{E}(130 \mathrm{mg}, 0.332 \mathrm{mmol})$ in $\mathrm{MeOH}(2.5 \mathrm{ml})$ was added $4 \mathrm{~N}$ HCl-EtOAc (2 $\mathrm{ml})$. The mixture was refluxed for $1 \mathrm{~h}$, cooled to room temperature and concentrated under reduced pressure to afford the crude product. To this flask was added dry THF (5.0 ml), Et $3 \mathrm{~N}$ (67.2 $\mathrm{mg}, 0.664 \mathrm{mmol})$, $p$-nitrophenyl palmitate $(250.7 \mathrm{mg}, 0.664 \mathrm{mmol}$ ) and DMAP (cat.) successively. The reaction mixture was stirred at room temperature for $10 \mathrm{~h}$ till no further change in reaction mixture composition as monitored by TLC. The reaction was quenched by adding saturated aqueous $\mathrm{NaHCO}_{3}$ and $1 \mathrm{~N} \mathrm{NaOH}$ solutions. The mixture was extracted with EtOAc $(3 \mathrm{x}$ 
$10 \mathrm{ml}$ ), washed with brine and dried over $\mathrm{Na}_{2} \mathrm{SO}_{4}$. The solvent was evaporated and residue was purified by column chromatography on silica gel, eluting with hexane/EtOAc (68:32) to yield pure 1c $(82.1 \mathrm{mg}, 54 \%)$ as a white solid; $\mathrm{mp} 95-97{ }^{\circ} \mathrm{C}$; $[\alpha]_{\mathrm{D}}{ }^{25}-3.6\left(c 1, \mathrm{CHCl}_{3}\right)$; IR $(\mathrm{KBr}) v_{\max }$ $\left(\mathrm{cm}^{-1}\right)$ 3460, 3305, 2964,1732, 1670, 1652, 1380; ${ }^{1} \mathrm{H}$ NMR (400 MHz, $\left.\mathrm{CDCl}_{3}\right): \delta 0.88(\mathrm{t}, J=7.0$ $\mathrm{Hz}, 3 \mathrm{H}), 1.25-1.33(\mathrm{~m}, 24 \mathrm{H}), 1.60-1.61(\mathrm{~m}, 2 \mathrm{H}), 2.20(\mathrm{t}, J=6.8 \mathrm{~Hz}, 2 \mathrm{H}), 4.31(\mathrm{~d}, J=6.2 \mathrm{~Hz}$, $1 \mathrm{H}), 4.40-4.45(\mathrm{~m}, 2 \mathrm{H}), 4.83(\mathrm{~s}, 1 \mathrm{H}), 5.93(\mathrm{~d}, J=7.3 \mathrm{~Hz}, 1 \mathrm{H}), 7.44-7.45(\mathrm{~m}, 3 \mathrm{H}), 7.51-7.56(\mathrm{~m}$, 2H), $8.08(\mathrm{~s}, 1 \mathrm{H}) \mathrm{ppm} ;{ }^{13} \mathrm{C} \mathrm{NMR}\left(100 \mathrm{MHz}, \mathrm{CDCl}_{3}\right): \delta 14.1,22.7,25.5,29.3,29.6,31.9$, 36.6, $46.5,64.3,64.9,125.1,129.0,130.2,133.6,148.8,164.9,173.2 \mathrm{ppm} . \mathrm{MS}, \mathrm{m} / z(\%)=458.6(\mathrm{M}+$ $1,18)^{+}, 480.6(\mathrm{M}+\mathrm{Na}, 55)^{+}$; Anal. Calcd for $\mathrm{C}_{28} \mathrm{H}_{43} \mathrm{NO}_{4}: \mathrm{C}, 73.48 ; \mathrm{H}, 9.47 ; \mathrm{N}, 3.06 \%$. Found: C, $73.39 ; \mathrm{H}, 9.44 ; \mathrm{N}, 3.04 \%$.

\section{Acknowledgements}

The authors are thankful to CSIR, New Delhi for providing financial assistance and GK and SK for fellowship. We are grateful to SAIF (Mr. Avtar Singh for NMR), Panjab University, Chandigarh for analytical facilities.

\section{References}

1. Basavaiah, D.; Rao, A. J.; Satyanarayana, T. Chem. Rev. 2003, 103, 811.

2. Dounay, A. B.; Overman, L. E. Chem. Rev. 2003, 103, 2945.

3. For the examples of natural products synthesized using the Baylis-Hillman reaction, see: (a) Basavaiah, D.; Rao, P. D.; Hyma, R. S. Tetrahedron 1996, 52, 8001. (b) Drewes, S. E.; Roos, G. H. P. Tetrahedron 1988, 44, 4653. (c) Iwabuchi, Y.; Furukawa, M.; Esumi, T.; Hatakeyama, S. Chem. Commun. 2001, 2030. (d) Iwabuchi, Y.; Sugihara, T.; Esumi, T.; Hatakeyama, S. Tetrahedron Lett. 2001, 42, 7867.

4. For the examples of natural products synthesized using the Heck reaction, see: (a) Sato, Y.; Mori, M.; Shibasaki, M. Tetrahedron:Asymmetry 1995, 6, 757. (b) Yoda, H.; Katoh, H.; Ujihara, Y.; Takabe, K. Tetrahedron Lett. 2001, 42, 2509. (c) Kondo, K.; Sodeoka, M; Shibasaki, M. J. Org. Chem. 1995, 60, 4322.

5. For the general review of Baylis-Hillman reaction, see: (a) Ciganek, E. In Organic Reactions; Paquette, L. A. Ed.; John Wiley \& Sons: New York, 1997; Vol. 51, pp 201-350. (b) Kim, J. N.; Lee, K. Y. Curr. Org. Chem. 2002, 6, 627. (c) Lee, K. Y.; Gowrisankar, S.; Kim, J. N. Bull. Korean Chem. Soc. 2005, 26, 1481. (d) Basavaiah, D.; Reddy, B. S.; Badsara, S. S. Chem. Rev. 2010, 110, 5447.

6. For recent examples of Baylis-Hillman reaction, see: (a) Xue, S.; He, L.; Han, K.-Z.; Liu, Y.-K.; Guo, Q.-X. Synlett 2005, 8, 1247. (b) Calmes, M.; Akkari, R.; Barthes, N.; Escale, F.; Martinez, J. Tetrahedron:Asymmetry 2005, 16, 2179. (c) Gowrisankar, S.; Lee, H. S.; Lee, 
K. Y.; Lee, J.-E.; Kim, J. N. Tetrahedron Lett. 2007, 48, 8619. (d) Lee, H. S.; Kim, S. H.; Kim, T. H.; Kim, J. N. Tetrahedron Lett. 2008, 49, 1773. (e) Gowrisankar, S.; Lee, H. S.; Kim, J. M.; Kim, J. N. Tetrahedron Lett. 2008, 49, 1670.

7. (a) Ameer, F.; Drewes, S. E.; Houston-McMillan, M. S.; Kaye, P. T. S. Afr. J. Chem. 1986, 39, 57. (b) Roos, G.; Manickum, T. Synth. Commum, 1991, 21, 2269. (c) Manickum, T.; Roos, G. H. P. S. Afr. J. Chem. 1994, 47, 1. (d) Nayak, S. P.; Thijs, L.; Zwanenburg Tetrahedron Lett. 1999, 40, 981. (e) Almeida, B. W. P.; Coelho, F. Tetrahedron Lett. 2003, 44, 937. (f) Krishna, P. R.; Sachwani, R.; Kannan, V. Chem. Commun. 2004, 2580. (g) Coelho, F.; Diaz, G.; Abella, C. A. M.; Almeida, W. P. Synlett 2006, 435. (h) Nakano, A.; Takahashi, K.; Ishihara, J.; Hatakeyama, S. Org. Lett. 2006, 8, 5357.

8. For reviews of Heck reaction, see: (a) Fu, G. C. Acc. Chem. Res. 2008, 41, 1555. (b) Alberico, D.; Scott, M. E.; Lautens, M. Chem. Rev. 2007, 107, 174. (c) Sabino, A. A.; Machado, A. H. L.; Correia, C. R.; Eberlin, M. N. Angew. Chem. Int. Ed. 2004, 43, 2514. (d) Beletskaya, I. P.; Cheprakov, A. V. Chem. Rev. 2000, 100, 3909.

9. Gowrisankar, S.; Lee, H. S.; Kim, S. H.; Lee, K. Y.; Kim, J. N. Tetrahedron 2009, 65, 8769.

10. (a) Kumareswaran, R.; Vanker, Y. D. Synth. Commun. 1998, 28, 2291. (b) Sunder, N.; Bhat, S. V. Synth. Commun. 1998, 28, 2311. (c) Basavaiah, D.; Muthukumaran, K. Tetrahedron 1998, 54, 4943. (d) Kulkarni, B. A.; Ganesan, A. J. Comb. Chem. 1999, 1, 373. (e) Kabalka, G, W.; Venkataiah, B.; Dong, G. Org. Lett. 2003, 5, 3803. (f) Perez, R.; Veronese, D.; Coelho, F.; Antunes, O. A. C. Tetrahedron Lett. 2006, 47, 1325. (g) Coelho, F.; Veronese, D.; Pavam, C. H.; De Paula, V. I.; Buffon, R. Tetrahedron 2006, 62, 4563. (h) Vasudevan, A.; Tseng, P.-S.; Djuric, S. W. Tetrahedron Lett. 2006, 47, 8591. (i) Lee, H. S.; Kim, S. H.; Gowrisankar, S.; Kim, J. N. Tetrahedron 2008, 64, 7183. (j) Ferreira, B. R. V.; Pirovani, R.V.; Souza-Filho, L. G.; Coelho, F. Tetrahedron 2009, 65, 7712.

11. Kim, J. M.; Kim, K, H.; Kim, T. H.; Kim J. N. Tetrahedron Lett. 2008, 49, 3248.

12. (a) Tani, M.; Ito, M.; Igarashi, Y. Cell. Signal. 2007, 19, 229. (b) Riethmuller, J.; Riehle, A.; Grassme, H.; Gulbins, E. Biochim. Biophys. Acta 2006, 1758, 2139. (c) Snook, C. F.; Jones, J. A.; Hannun, Y. A. Biochim. Biophys. Acta 2006, 1761, 927. (d) EI Alwani, M.; Wu, B. X.; Obeid, L. M.; Hannun, Y.A. Pharmacol. Ther. 2006, 112, 171.

13. Pettus, B, J.; Chalfant, C, E.; Hannun, Y. A. Biochim. Biophys. Acta 2002, 1585, 114.

14. (a) Bourbon, N. A.; Sandirasegarane, L.; Kester, M. J. Biol. Chem. 2002, 277, 3286. (b) Webb, L. M.; Arnholt, A. T.; Venable, M. E. Mol. Cell Biochem. 2010, 337, 153.

15. Modrak, D. E.; Leon, E.; Goldenberg, D. M.; Gold, D. V. Mol. Cancer Res. 2009, 7, 890.

16. (a) Hannum, Y. A.; Luberto, C. Trends Cell Biol. 2000, 10, 73. (b) Dobrowsky, R. T.; Kolesnick, R. N. Methods Cell Biol. 2001, 66, 135. (c) Ruvolo, P. P. Leukemia 2001, 15, 1153.

17. (a) Kim, K.; Kang, J.; Kim, S.; Choi, S.; Lim, S.; Im. C.; Yim, C. Arch. Pharm. Res. 2007, 30, 570. (b) Liu, J.; Antoon, J. W.; Ponnapakkam, A.; Beckman, B. S.; Foroozesh, M. Bioorg. Med. Chem. Lett. 2010, 18, 5316-5322.

18. Wascholowski, V.; Giannis, A. Angew. Chem. Int. Ed. 2006, 45, 827. 
19. For the review on ceramide and its derivatives see: (a) Koskinen, P. M.; Koskinen, A. M. P. Synthesis 1998, 1075. (b) Curfman, C.; Liotta, D. Methods Enzymol. 1999, 311, 391. (c) Merrill, A. H. Jr.; Hannum, Y. A. Methods Enzymol 2000, 311, 91. (d) Liao, J.; Tao, J.; Lin, G.; Liu, D. Tetrahedron 2005, 61, 4715. (e) Park, J.-J.; Lee, J. H.; Li, Q.; Diaz, K.; Chang, Y.-T.; Chung, S.-K. Bioorg. Chem. 2008, 36, 220.

20. For selected publications on recent synthesis of ceramide and its analogues see: (a) Mathew, T.; Cavallari, M.; Billich, A.; Bornancin, F.; Nussbaumer, P.; De Libero, G.; Vasella, A. Chem. Biodivers. 2009, 6, 1688. (b) Bai, A.; Szulc, Z. M.; Bielawski, J.; Mayroo, N.; Liu, X.; Norris, J.; Hannun, Y. A.; Bielawska, A. Bioorg. Med. Chem. 2009, 17, 1840. (c) Antoon, J. W.; Liu, J.; Gestaut, M. M.; Burow, M. E.; Beckman, B. S.; Foroozesh, M. J. Med. Chem. 2009, 52, 5748. (d) Antoon, J. W.; Liu, J.; Ponnapakkam, A. P.; Gestaut, M. M.; Foroozesh, M.; Beckman, B. S. Cancer Chemother. Pharmacol. 2010, 65, 1191.

21. Drewes, S. E.; Khan, A. A.; Rowland, K. Synth. Commun. 1993, 23, 183.

22. Chun, J.; He, L.; Byun, H.-S.; Bittman, R. J. Org. Chem. 2000, 65, 7634.

23. Liang, X.; Andersch, J.; Bols, M. J. Chem. Soc., Perkin Trans.1 2001, 2136.

24. (a) Dondoni, A.; Perrone, D. Synthesis 1997, 527. (b) Williams, L.; Zhang, Z.; Shao, F.; Carroll, P. J.; Joullie, M. M. Tetrahedron 1996, 52, 11673. (c) Yonezawa, Y.; Shimizu, K.; Yoon, K.-S.; Shin, C.- G. Synthesis 2000, 634.

25. Kumar, G.; Kaur, S.; Singh, V. Helv. Chim. Acta 2010, DOI: 10.1002/hlca.201000277.

26. (a) Cherest, M.; Felkin, H.; Prudent, N. Tetrahedron Lett. 1968, 18, 2199. (b) Anh, N. T. Top. Curr. Chem. 1980, 88, 144. (c) Hoffman, R. W. Chem. Rev. 1989, 89, 1841.

27. Nakamura,Y.; Matsubara, R.; Kitagawa, H.; Kobayashi, S.; Kumagai, K.; Yasuda, S.; Hanada, K. J. Med. Chem. 2003, 46, 3688. 